\title{
Measurement of left ventricular volumes and function using O-15-labeled carbon monoxide gated PET
}

\author{
Philipp A. Kaufmann, MD
}

\section{See related article, p. 639}

Although single photon emission computed tomography (SPECT) has been the cornerstone of noninvasive assessment of coronary artery disease (CAD) and myocardial perfusion imaging (MPI) for many years, MPI now increasingly also involves positron emission tomography (PET). In many centers equipped with PET facilities, it provides a well-established technique that is routinely used for the noninvasive imaging and quantification of myocardial perfusion and metabolism.

As PET has inherently implemented attenuation correction, the accuracy of PET is expected to be superior to that of SPECT. The recent introduction of gated SPECT, however, has further increased the value of SPECT MPI. ${ }^{1}$ In fact, end-diastolic and end-systolic left ventricular (LV) volume and ejection fraction (EF) are important predictors of prognosis and death in patients with cardiac disease, particularly after myocardial infarction but also in nonischemic cardiac disease. Furthermore, parameters of regional and global LV function are used to evaluate the efficacy of revascularization in patients with CAD and chronic but reversible LV dysfunction. Assessment of functional LV parameters provides additional information, which substantially increases the clinical impact of MPI. As no additional radiation dose needs to be applied to get this information, it should become the clinical standard to assess function by use of electrocardiographic triggering. The old and still valid rule that radiation dose should be kept as low as reasonably achievable (ALARA) should now be completed by the statement on clinical information-to obtain as much as reasonably achievable (AMARA). Similar to the development in gated SPECT, which has

From the Cardiovascular Center, Nuclear Cardiology, University Hospital Zurich, Zurich, Switzerland.

Reprint requests: Philipp A. Kaufmann, MD, Swiss National Science Foundation, Nuclear Medicine and Cardiology, University Hospital Zurich, Zurich, Switzerland; pak@usz.ch.

J Nucl Cardiol 2005;12:620-1.

$1071-3581 / \$ 30.00$

Copyright $(9) 2005$ by the American Society of Nuclear Cardiology. doi:10.1016/j.nuclcard.2005.08.004 brought a large number of validation studies for each quantification method, this has now to be repeated by the PET community. Consequently, different studies have validated the measurement of LV volumes by electrocardiography-gated PET by use of different myocardial tracers such as fluorine 18 fluorodeoxyglucose $\mathrm{e}^{2-4}$ or nitrogen 13 ammonia., ${ }^{5,6}$ For the blood pool tracer oxygen 15-labeled carbon dioxide (CO), only limited data from small pilot studies exist, in which the protocol was specifically chosen to measure LV volumes by gating. ${ }^{7-9}$

In this issue of the Journal of Nuclear Cardiology, Hofman et $\mathrm{al}^{10}$ provide evidence that LV volumes and EF can be reliably measured noninvasively by means of gated PET with the use of myocardial blood pool images routinely acquired for use in perfusion quantification. This contributes to the above-mentioned aim to obtain as much information as reasonably achievable with any application of any radiotracer. The authors did so with $2000 \mathrm{MBq} \mathrm{O}-15-$ labeled CO, resulting in an effective dose equivalent of as low as around $0.3 \mathrm{mSv}$. This certainly meets the criterion of ALARA, as it is lower by about one order as compared with previous reports. ${ }^{7,9}$ Measurements were performed on a PET scanner and compared with the gold standard, magnetic resonance imaging.

Overall, despite a substantially shortened acquisition time and lower dose, correlation was very good between PET and magnetic resonance imaging for end-systolic $(r$ $=0.90)$ and end-diastolic LV volumes $(r=0.096)$ with reasonable limits of agreement, although correlation was slightly inferior for LV EF $(r=0.86)$. Similar to previous reports, ${ }^{7} \mathrm{LV}$ EF tended to be underestimated. The fact that EF was not measured on the same day by the two different techniques is probably not the main reason for these differences. It is much more plausible that, inherently, two different techniques may not measure exactly the same volume, for example, because of difficulties in identifying the basal (mitral valve) plane. It is, therefore, very important to know the normal values for each single technique with its specific equipment and isotope, and the present study is helpful in establishing this knowledge for the use of O-15-labeled CO PET scans. At present, a major drawback is that each institution is using its own software, and therefore results 
cannot be directly compared between different sites. Standardization of gated SPECT measurement provided the base to its overwhelming success. Similarly, standardization of gated PET data acquisition will advance acceptance and importance of this precious technique.

\section{Acknowledgment}

The author has indicated he has no financial conflicts of interest.

\section{References}

1. DePuey EG, Heller G, Taillefer R. Clinical application of gated myocardial perfusion SPECT. In: DePuey EG, Garcia CE, Berman D, editors. Cardiac SPECT imaging. 2nd ed. Philadelphia: Lippincott Williams \& Wilkins; 2001. p. 211-30.

2. Slart RH, Bax JJ, de Jong RM, de Boer J, Lamb HJ, Mook PH, et al. Comparison of gated PET with MRI for evaluation of left ventricular function in patients with coronary artery disease. J Nucl Med 2004;45:176-82.

3. Schaefer WM, Lipke CS, Nowak B, Kaiser HJ, Reinartz P, Buecker A, et al. Validation of QGS and 4D-MSPECT for quantification of left ventricular volumes and ejection fraction from gated 18F-FDG PET: comparison with cardiac MRI. J Nucl Med 2004;45:74-9.
4. Khorsand A, Graf S, Frank H, Kletter K, Sochor H, Maurer G, et al. Model-based analysis of electrocardiography-gated cardiac (18)F-FDG PET images to assess left ventricular geometry and contractile function. J Nucl Med 2003;44:1741-6.

5. Hickey KT, Sciacca RR, Bokhari S, Rodriguez O, Chou RL, Faber TL, et al. Assessment of cardiac wall motion and ejection fraction with gated PET using N-13 ammonia. Clin Nucl Med 2004;29: 243-8.

6. Okazawa H, Takahashi M, Hata T, Sugimoto K, Kishibe Y, Tsuji T. Quantitative evaluation of myocardial blood flow and ejection fraction with a single dose of (13) $\mathrm{NH}(3)$ and gated PET. J Nucl Med 2002;43:999-1005.

7. Rajappan K, Livieratos L, Camici PG, Pennell DJ. Measurement of ventricular volumes and function: a comparison of gated PET and cardiovascular magnetic resonance. J Nucl Med 2002;43:806-10.

8. Miller TR, Wallis JW, Landy BR, Gropler RJ, Sabharwal CL. Measurement of global and regional left ventricular function by cardiac PET. J Nucl Med 1994;35:999-1005.

9. Boyd HL, Gunn RN, Marinho NV, Karwatowski SP, Bailey DL, Costa DC, et al. Non-invasive measurement of left ventricular volumes and function by gated positron emission tomography. Eur J Nucl Med 1996;23:1594-602.

10. Hofman AH, Knaapen P, Boellaard R, Bondarenko O, Götte MJW, van Dockum WG, et al. Measurement of left ventricular volumes and function with O-15-labeled carbon monoxide gated positron emission tomography: comparison with magnetic resonance imaging. J Nucl Cardiol 2005;12:620-1. 